\title{
Application of Fuzzy Synchronization in the NLOS UV Communication System
}

\author{
G.S. Vasilyev ${ }^{1}$, O.R. Kuzichkin ${ }^{1}$, D.I. Surzhik ${ }^{2}$, I.S. Konstantinov ${ }^{1,3}$, S.A.Lazarev ${ }^{1}$ \\ ${ }^{1}$ Belgorod State University, Belgorod, 308015, Russia. \\ ${ }^{2}$ Vladimir State University, Vladimir, 600000, Russia. \\ ${ }^{3}$ Russian Timiryazev State Agrarian University, Moscow, 127550, Russia.
}

\begin{abstract}
To ensure the effective operation of UV-C communication systems in the non-line-of-sight (NLOS) mode, synchronization between an optical transmitter and an optical receiver is often required. The complex noise-like nature of the signal on the receiving side of the UV-C communication system determines the relevance of using various methods for synchronizing UV-C communication systems based on the neuro-fuzzy approach. This nature of the signal arises due to the strong attenuation of UV radiation in the atmosphere, the effects of intersymbol interference, and the instability of the signal propagation characteristics. The structural diagrams of the optical transmitter and optical receiver of the UV-C communication system are considered. The synthesis of the fuzzy controller of the UV communication system receiver was performed using the Takagi-Sugeno fuzzy inference algorithm; the last was selected due to lower computational costs compared to the Mamdani scheme. An artificial neural network with one hidden layer, trained by the error backpropagation algorithm was used to adjust the parameters of the fuzzy controller's membership functions. The modelling of the process for synchronization between the transmitter and the receiver in the NLOS UV communication system is performed. 16-position pulse-phase type of modulation (16-PPM) was chosen; an M-sequence with a length of 32 bits was used as a pseudo-random sequence. The modelling showed acceptable convergence of the algorithm and the effectiveness of the proposed fuzzy synchronization method in the NLOS UV communication system.
\end{abstract}

Keywords: NLOS UV communication, synchronization, fuzzy logic, Takagi-Sugeno algorithm, artificial neural network.

\section{INTRODUCTION}

The use of optical communication systems in the UV-C spectrum range from $200 \mathrm{~nm}$ to $280 \mathrm{~nm}$ is an attractive alternative to traditional radio communication systems, as well as optical systems of other spectral ranges (infrared, visible and soft UV with a wavelength of more than $280 \mathrm{~nm}$ ) [1-3 ]. The key advantages of UV-C communication systems are exhibited in conditions such as the lack of direct visibility between an optical transmitter and an optical receiver (nonline-of-sight, NLOS mode) due to the presence of high obstacles, the strong influence of natural electromagnetic interference, or deliberate suppression by means of electronic warfare. A review of the experimental setups of various authors showed that the use of synchronization between an optical transmitter and an optical receiver is often required to ensure the effective operation of NLOS UV communication systems [4, 5]. Traditional approaches aimed at ensuring synchronization of signals with pronounced fronts for NLOS UV communication systems may not be applicable. The complex noise-like nature of a signal on the receiving side of the UV-C communication system, which arises due to the strong attenuation of UV radiation in the atmosphere, and also effects of intersymbol interference, and instability of the signal propagation characteristics determine the relevance of the methods for the application of synchronisation in UV-C communication systems. The synchronisation is based on the neuro-fuzzy approach tested for chaotic signals, on artificial neural networks [6-8], genetic algorithms [9, 10] and fuzzy logic [11-14].

The aim of the work is to substantiate the use of neuro-fuzzy synchronization in the NLOS UV communication system.

\subsection{Block diagram of a synchronous NLOS UV communication system}

A typical block diagram of the NLOS UV communication system with analogue and digital receiving channels is shown in Fig. 1 [5]. The transmitting part of the system is represented by a pulse delay generator, a UV laser and a lens (instead of a UV laser, a UV-C single LED or an array of LEDs can also be used as an emitter). The receiving side of the system is represented by a collecting UV lens, a sun-blind filter and a photomultiplier tube (PMT), as well as analogue and digital receiving channels. This scheme uses the synchronization of signals at the transmitting and receiving sides based on the global positioning system (GPS). It should be noted that synchronisation is used both in the analogue mode for receiving signal photons (analogue receiving channel consisting of a high-speed preamplifier and an oscilloscope), and in the digital receiving channel (photon counter). 


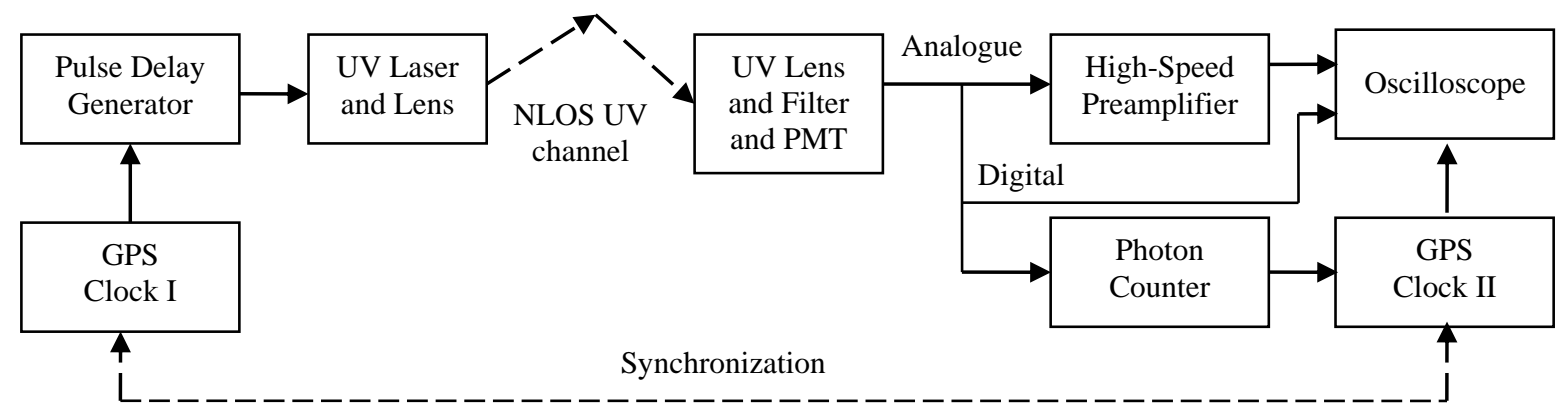

Fig. 1: Block diagram of the synchronous NLOS UV communication system

Ensuring effective synchronization presents significant difficulties due to the complex pseudo-random nature of the signal at the receiving side of the NLOS UV communication system. This arises due to strong attenuation of UV radiation in the atmosphere, effects of intersymbol interference, and instability of the signal propagation characteristics. Traditional approaches focused on providing synchronization of signals with pronounced edges may not be applicable to the NLOS UV communication system. Therefore, unconventional approaches to synchronizing NLOS UV communication systems are relevant, which are tested for chaotic wide spectrum signals and are based on artificial neural networks [6-8], genetic algorithms [9, 10] and fuzzy logic [11-14].

\subsection{Fuzzy controller for a synchronization subsystem of the NLOS UV communication system}

Stationary and especially mobile communication centres impose very stringent requirements for weight and size and energy parameters; this leads to a limitation of the computing resources used. Therefore, for the implementation of the synchronization subsystem of the NLOS UV communication system based on fuzzy logic, the least laborious fuzzy inference algorithms are promising. Therefore, it is advisable to use the Takagi-Sugeno algorithm, which differs from the Mamdani algorithm in less laborious and simple description of complex systems with a large number of elements and the relationships between them $[15,16]$.

The error of synchronization between the optical transmitter and the optical receiver of the NLOS UV communication system is defined as the difference of two signals:

$Y_{e}(t)=\hat{Y}(t)-Y(t)=\left[e_{1}(t), e_{2}(t), \ldots, e_{\delta}(t)\right]^{\mathrm{T}}$.

The fuzzy controller is defined by the following expressions:

IF $e_{1}(t)$ is $M_{l 1}$ and $\ldots$ and $e_{\delta}(t)$ is $M_{l \delta}$ THEN $U(t)=-K_{l} Y_{e}(t)$, (2)

Where $l=1,2, \ldots, m$ - logical controller rule number, $M_{l \eta}(\eta=$ $1,2, \ldots, \delta)$ - fuzzy sets.

The result of defuzzification by the centre of gravity method for one-point sets is the fuzzy controller relation

$$
U(t)=\frac{\sum_{l=1}^{m} w_{l}(t) K_{l} Y_{e}(t)}{\sum_{l=1}^{m} w_{l}(t)}=-\sum_{l=1}^{m} \bar{h}_{l}(t) K_{l} Y_{e}(t)
$$

Where $w_{l}(t)=\prod_{\eta=1}^{\delta} M_{l \eta}\left(e_{\eta}(t)\right), M_{l \eta}\left(e_{\eta}(t)\right)$ - grade of membership of $e_{\eta}(t)$ in $\quad M_{l \eta} ;$ conditions $\bar{h}_{l}(t)=\frac{w_{l}(t)}{\sum_{l=1}^{m} w_{l}(t)}$ и $\sum_{l=1}^{m} \bar{h}_{l}(t)=1$ are satisfied for every $t$.

Setting the parameters of membership functions is a complex nonlinear programming problem, the solution of which is carried out on the basis of various mathematical methods, for example, the apparatus for artificial neural networks (NN) [6$8,17]$.

\subsection{Modelling of the NLOS UV channel and communication system}

The scattering model characterizing the probability of photon scattering in a given direction, is described by the phase scattering function. The phase function is the weighted sum of the phase functions of Rayleigh molecular scattering and Mie aerosol scattering [18, 19],

$$
\begin{aligned}
& p^{R}(\mu)=\frac{3\left[1+3 \gamma+(1-\gamma) \mu^{2}\right]}{16 \pi(1+2 \gamma)}, \\
& p^{M}(\mu)=\frac{1-g^{2}}{4 \pi}\left[\frac{1}{\left(1+g^{2}-2 g \mu\right)^{3 / 2}}+f \frac{0.5\left(3 \mu^{2}-1\right)}{\left(1+g^{2}\right)^{3 / 2}}\right],
\end{aligned}
$$

Where $\mu=\cos \theta_{s}$ - scattering angle cosine, $\gamma, g, f-$ parameters of the scattering model. Scattering weights $k_{s}^{R a y}$ and $k_{s}^{M i e}$, as well as the absorption coefficient $\mathrm{k}_{\mathrm{a}}$ are determined by the radiation wavelength according to table 1 . 
International Journal of Engineering Research and Technology. ISSN 0974-3154, Volume 13, Number 11 (2020), pp. $3825-3829$

(C) International Research Publication House. https://dx.doi.org/10.37624/IJERT/13.11.2020.3825-3829

Table 1: Scattering and absorption coefficients for various radiation wavelengths [19]

\begin{tabular}{|c|c|c|c|}
\hline $\begin{array}{c}\text { Wavelength } \lambda, \\
\mathrm{nm}\end{array}$ & $k_{s}^{\text {Ray }}\left(\mathrm{km}^{-1}\right)$ & $k_{s}^{\text {Mie }}\left(\mathrm{km}^{-1}\right)$ & $k_{a}\left(\mathrm{~km}^{-1}\right)$ \\
\hline 230 & 0.493 & 0.623 & 2.581 \\
\hline 240 & 0.406 & 0.531 & 1.731 \\
\hline 250 & 0.338 & 0.421 & 1.202 \\
\hline 260 & 0.266 & 0.284 & 0.802 \\
\hline 270 & 0.241 & 0.277 & 0.621 \\
\hline 280 & 0.194 & 0.272 & 0.322 \\
\hline 290 & 0.177 & 0.266 & 0.046 \\
\hline 300 & 0.145 & 0.261 & 0.039 \\
\hline 310 & 0.132 & 0.234 & 0.005 \\
\hline
\end{tabular}

In difficult weather conditions, the Mie model additionally includes terms describing the contribution of certain types of aerosol particles with different radii and concentrations [20, 21]. The geometric parameters of the NLOS UV channel, which determine the properties of radiation propagation, are: the distance between the optical transmitter and the optical receiver (communication range), the elevation angles (site angles) of the transmitter and receiver, the width of the radiation patterns of the transmitter and receiver, and the aperture area of the receiver. The attenuation and distortion of a signal in the UV channel are caused by scattering and absorption of photons, as well as the pulse widening in the NLOS UV channel due to different distances travelled by individual scattered photons. The pulse widening is critical for investigating intersymbol interference in UV communication systems. Analytical models of the UV channel are of little use for modelling of these effects under various operating conditions (for example, for studying multiple scattering) [22, 23]. Therefore, it is advisable to use statistical modelling of UV channels based on the Monte Carlo method [24-26].

\section{MODELLING RESULTS}

The modelling of the process on synchronization between the optical transmitter and optical receiver of the NLOS UV communication system is performed. The following values of the UV channel parameters were taken: communication range $\mathrm{r}=100 \mathrm{~m}$, the elevation angles of the transmitter and receiver $\theta 1=30^{\circ}$ and $\theta 2=50^{\circ}$, the width of the transmitter and receiver patterns $\varphi 1=10^{\circ}$ and $\varphi 2=30^{\circ}$, the radiation wavelength $\lambda=260 \mathrm{~nm}$, the scattering and absorption coefficients are those for clear weather, the receiver aperture area $\mathrm{Ar}=1.77 \mathrm{~cm}^{2}$. The optical transmitter power was assumed to be equal $50 \mathrm{~mW}$, the bit rate was $100 \mathrm{kbps}$., 4position pulse-phase modulation type (16-PPM) was chosen; it has a higher energy efficiency compared to on-off keying (OOK) [27]; The 16-PPM timing diagram is shown in Fig. 2.

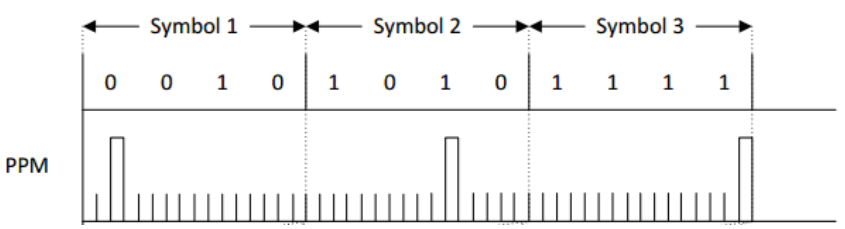

Figure 2: Timing diagram of 16-PPM modulation

A 32-bit pseudo-random M-sequence was taken as the transmitted sequence. The time interval for calculating transients was chosen to be equal $5 \mu$ s. To train the fuzzy logic controller, a neural network with one hidden layer was used. Neural network training was performed using the back propagation algorithm. The following objective function was used as a criterion for the training quality

$$
J=1 / \sum_{k}\left(e_{1}(k)^{2}+e_{2}(k)^{2}\right),
$$

Where $\mathrm{k}$ is the reference number, $\mathrm{e}_{1}(\mathrm{k})$ is the synchronization error (1); $\mathrm{e}_{2}(\mathrm{k})$ is the error derivative. Higher error derivatives were not used. The dependence diagram for the normalized error of the neural fuzzy synchronization system on the reference number $\mathrm{k}$ obtained during training of the neural network is shown in Fig. 3. As we can see from the graph, training is completed in about 50 counts.

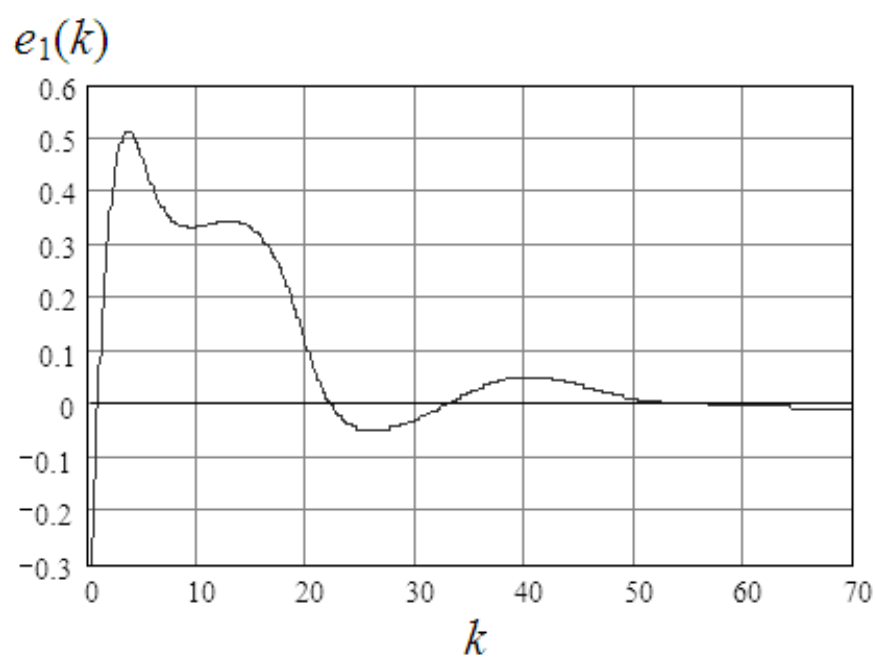

Fig. 3: Neuro-fuzzy system synchronization error of the NLOS UV communication system during training

\section{CONCLUSION}

Ensuring the effective operation of NLOS UV communication systems often requires the use of synchronization between an optical transmitter and an optical receiver. The use of neurofuzzy synchronization has been tested to detect a highly noisy signal on the receiving side of the UV-C system. A fuzzy controller for the NLOS UV communication system based on the Takagi-Sugeno fuzzy inference algorithm was synthesized. The parameters of the fuzzy controller are 
adjusted using an artificial neural network with one hidden layer trained using the algorithm for back propagation of error. Modelling of the process for synchronization between the optical transmitter and optical receiver of the NLOS UV communication system has been carried out; it has confirmed that the algorithm converges acceptably and the proposed fuzzy synchronization method in the NLOS UV communication system is effective.

\section{ACKNOWLEDGMENTS}

This work was supported by the Ministry of Science and Higher Education of the Russian Federation under Grant RFMEFI57518X0175 "Development of digital communication modules of mobile devices operating on the basis of UV data channels for establishing special purpose wireless ad-hoc networks".

\section{REFERENCES}

[1] Z. Xu and B. M. Sadler, "Ultraviolet communications: Potential and state-of-the-art," IEEE Commun. Mag., vol. 46, no. 5, pp. 67-73, May 2008

[2] R. J. Drost and B. M. Sadler, "Survey of ultraviolet non-line-of-sight communications,"Semicond. Sci. Technol., vol. 29, no. 8, Aug. 2014, Art. no. 084006.

[3] D. M. Reilly, D. T. Moriarty, and J. A. Maynard, "Unique properties of solar blind ultraviolet communication systems for unattended ground-sensor networks," in Proc. SPIE, vol. 5611. London, U.K., 2004, pp. 244-254

[4] L. Liao, G. Chen, B. M. Sadler, and Z. Li, "GPS synchronized UV communication system performance based on USRP," inProc. SPIE, vol. 8874. San Diego, CA, USA, Aug. 2013, pp. 1-8

[5] Drost, Robert \& Lang, Tian \& Chen, Gang \& Liao, Linchao \& Li, Zening \& Sadler, B.M.. (2015). Longdistance non-line-of-sight ultraviolet communication channel analysis: Experimentation and modelling. IET Optoelectronics. 9. 10.1049/iet-opt.2014.0121.

[6] M. Liu, "Optimal exponential synchronization of general chaotic delayed neural networks: an LMI approach," Neural Networks, vol. 22, pp. 949-957, 2009.

[7] D. Qi, M. Liu, M. Qiu and S. Zhang, "Exponential Hळ synchronization of general discrete-time chaotic neural networks with or without time delays," IEEE Trans. Neural Network, vol. 21, pp. 1358-1365, 2010.

[8] H. R. Karimi and H. Gao, "New delay-dependent exponential $\mathrm{H} \infty$ synchronization for uncertain neural networks with mixed time delays," IEEE Trans. Syst. Man Cybern. B: Cybern., vol. 40, no. 1, pp. 173-185, Feb. 2010.

[9] C. C. Sun, H. Y. Chung and W. J. Chang, "Design the T-S fuzzy controller for a class of T-S fuzzy models via genetic algorithm," IEEE Int. Conf. on Fuzzy Systems, vol. 1, pp. 278-283, 2002.

[10] Babaei Pourkargar, Davood \& Shahrokhi, Mohammad. (2011). Optimal Fuzzy Synchronization Of Generalized Lorenz Chaotic Systems. The Journal of Mathematics and Computer $\quad$ Science. 22. 27-36. 10.22436/jmcs.002.01.04.

[11] H. K. Lam, W. K. Ling, H. H. C. Iu and S. S. H. Ling, "Synchronization of chaotic systems using timedelayed fuzzy state-feedback controller," IEEE Trans. Circuit and Systems I, vol. 55, pp. 893-903, 2008.

[12] K. R. Lee, J. H. Kim, E. T. Jeung and H. B. Park, "Output feedback robust $\mathrm{H} \infty$ control of uncertain fuzzy dynamic systems with time-varying delay," IEEE Trans. Fuzzy Systems, vol. 8, pp. 657-664, 2000.

[13] B. S. Chen, C. H. Chiang and S. K. Nguang, "Robust $\mathrm{H} \infty$ Synchronization design of nonlinear coupled network via fuzzy interpolation method," IEEE Trans. on Circuits and Systems I, vol. 58, pp. 349-362, 2011.

[14] Ha, Shumin \& Liu, Heng \& Li, Shenggang \& Liu, Aijing. (2019). Backstepping-Based Adaptive Fuzzy Synchronization Control for a Class of FractionalOrder Chaotic Systems with Input Saturation. International Journal of Fuzzy Systems. 21. 10.1007/s40815-019-00663-5.

[15] T. Takagi and M. Sugeno, "Fuzzy identification of systems and its applications to modeling and control," IEEE Trans. Systems, Man, and Cybernetics, vol. 15, pp. 116-132, 1985.

[16] Decision support systems: textbook and workshop for undergraduate and graduate programs / V. G. Khalin [et.al.]; edited by V. G. Khalin, G. V. Chernovaya. Moscow: Yurayt Publishing House, 2019 .- 494 p.

[17] S. Limanond, and J. Si, "Neural-network-based control design: An LMI approach," IEEE Trans. Neural Networks, vol. 9, pp. 1422-1429, 1998.

[18] Bohren G. F. and D. R. Huffman, Absorption and scattering by a sphere, Absorption and scattering of light by small particles (Wiley, 1983).

[19] Ding H., Chen G., Majumdar A., Sadler B. and Xu Z. Modeling of non-line-of-sight ultraviolet scattering channels for communication IEEE J. Sel. Areas Commun. 271535-44, 2009.

[20] Xu C., Zhang H., and Cheng J. Effects of haze particles and fog droplets on NLOS ultraviolet communication channels Vol. 23, Iss. 18, pp. 23259-23269 (2015).

[21] I.S. Konstantinov, G.S. Vasilyev, O.R. Kuzichkin, I.A. Kurilov, S.A. Lazarev. Modeling and Analysis of the Characteristics of Ultraviolet Channels under Different Conditions of Radiation Propagation for the Organization of Wireless AD-HOC Network // JARDCS - Journal of Advanced Research in Dynamical and Control Systems - 2018. - 07-Special Issue, pp. 
http://jardcs.org/abstract.php?archiveid=5147.

[22] H. M. R. Luettgen, J. H. Shapiro, and D. M. Reilly, "Non-line-of-sight single-scatter propagation model," J. Opt. Soc. Amer. A, Opt. Image Sci., vol. 8, no. 12, pp. 1964-1972, Dec. 1991

[23] Yin et al., "Analytical model of non-line-of-sight single-scatter propagation, "J. Opt. Soc. Amer. A, Opt. Image Sci., vol. 27, no. 7, pp. 1505-1509, Jul. 2010.

[24] R. J. Drost, T. J. Moore, and B. M. Sadler, "MonteCarlo-based multiple-scattering channel modeling for non-line-of-sight ultravio-let communications," in Proc. SPIE, vol. 8038. Orlando, FL, USA, Apr. 2011, Art. no. 803802 .

[25] Konstantinov I.S., Vasilyev G.S., Kuzichkin O.R., Surzhik D.I., Lazarev S.A. Numerical and Analytical Modeling of Wireless UV Communication Channels for the Organization of Wireless Ad-Hoc Network // IJCSNS - International Journal of Computer Science and Network Security - 2018. - Vol. 18, No. 8, pp. 98104. Доступ:

http://paper.ijcsns.org/07_book/201808/20180815.pdf (open access).

[26] Igor S. Konstantinov, G. S. Vasyliev, Oleg R. Kuzichkin, D.I. Surzhik, I. A. Kurilov, Sergey A. Lazarev. Development Of UV Communication Channels Characteristics Modeling Algorithm In A Mobile Ad-Hoc Network / Journal of Advanced Research in Dynamical and Control Systems (JARDCS) / ISSN: 1943-023X / Volume 11 | 08Special Issue, 2019. Pages: 1920-1928. http://www.jardcs.org/abstract.php?id=2543

[27] Elganimi T.Y. Performance Comparison between OOK, PPM and PAM Modulation Schemes for Free Space Optical (FSO) Communication Systems: Analytical Study. International Journal of Computer Applications 79(11):22-27, October 2013. 